\title{
REPRESENTING FINITELY ADDITIVE INVARIANT PROBABILITIES ${ }^{1}$
}

\section{By Richard A. Olshen \\ Stanford University}

1. Introduction. Hewitt and Savage [6] have shown that finitely additive exchangeable probabilities on a product space are integral averages of power product probabilities. They prove this result as a corollary to their theorems on the countably additive case. This note adapts their technique to the study of more general invariant probabilities. From results of Farrell [4] and Choquet and Feldman ([7], Section 10) it is concluded that finitely additive invariant probabilities are averages of finitely additive ergodic probabilities.

In a countably additive context it seems necessary to impose restrictions on the Borel field being studied and on the maps used to define invariance and ergodicity. Relaxing the assumptions of one type must be balanced by strengthening those of the other (in addition to [4] and [7], see [1] and [12]). Here, however, the field of sets can be arbitrary, and the maps are assumed only to be measurable. Rather than state a host of theorems which can be proved, one particular case is proved in detail. Later on it is explained how the techniques can be applied to other problems. Several definitions of ergodicity are proposed and related to the one used. The final section contains a subjective probability interpretation of invariance and ergodicity.

2. A representation theorem. A homomorphism from one field of sets to another is a map which preserves finite unions, finite intersections, and complements. The notions of isomorphism and automorphism are defined in the obvious ways. A $\sigma$-homomorphism (isomorphism, automorphism) in addition preserves countable unions and intersections.

Assume $\Omega$ is a set, $\mathcal{F}$ a field of subsets of $\Omega$, and $T$ a $1-1$ bi-F-measurable map of $\Omega$ onto $\Omega$. T and its powers can be viewed as automorphisms of $\mathcal{F}$. A finitely additive probability $\mu$ on $\mathcal{F}$ is said to be invariant if $\mu(A)=\mu\left(T^{-1} A\right)\left[=\mu\left(T^{n} A\right)\right.$, $n= \pm 1, \pm 2 \cdots]$ for all $A \varepsilon \mathcal{F} ; \mu$ is ergodic if there do not exist $\delta>0$ and $A_{1}, A_{2}, \cdots \varepsilon \mathcal{F}$ for which $\delta<\mu\left(A_{n}\right)<1-\delta, \lim _{m, n \rightarrow \infty} \mu\left(A_{n} \triangle A_{m}\right)=0$, and $\mu\left(A_{n} \triangle T^{-1} A_{n}\right) \rightarrow 0$ ( $\triangle$ denotes symmetric difference). Let $\mathscr{g}(\mathcal{F})[\mathcal{E}(\mathcal{F})]$ be the set of finitely additive invariant [ergodic] probabilities on $\mathcal{F}$ and let $\beta$ be the smallest $\sigma$-field of subsets of $\mathcal{E}(\mathcal{F})$ containing all sets of the form $\{\nu \mid \nu \varepsilon \mathcal{E}(\mathcal{F})$, $\nu(A) \leqq \alpha\}$, where $0 \leqq \alpha \leqq 1$, and $A$ is a fixed set in $\mathcal{F}$.

(1) Theorem. $\mathscr{G}(\mathcal{F})$ is not empty. For each $\mu \in \mathcal{G}(\mathfrak{F})$ there is a unique countably additive probability $\lambda$ on $B$ satisfying

$$
\mu(A)=\int \varepsilon(\mathfrak{F}) \nu(A) d \lambda(\nu)
$$

Received 11 March 1968.

1 Prepared with partial support of U.S. Army Research Office Grant DA-ARO(D)-31124-G726. 
for all $A \varepsilon \mathcal{F}$. (It is clear that, conversely, if for a finitely additive probability $\mu$ on $\mathcal{F}$ there exists a $\lambda$ on $B$ satisfying (2) for all $A \in \mathcal{F}$, then $\mu \in \mathcal{G}(\mathcal{F})$.

Proof. By Stone's representation theorem for Boolean algebras ([11], Section 8) there is an isomorphism $\varphi$ of $\mathcal{F}$ onto the field $\mathcal{O}$ of compact open subsets of some compact, totally disconnected Hausdorff space $S$. Call $S$ "the" Stone space of $\mathfrak{F}$; any two such are homeomorphic. For $B \varepsilon \mathcal{O}$ define $U B=\varphi\left(T^{-1}\left(\varphi^{-1} B\right)\right)$. Of course $U$ and its powers are automorphisms of $\mathcal{O}$. Moreover, there exists a homeomorphism $\psi$ of $S$ with itself for which $\psi^{-1} B=U B$ for all $B \varepsilon \mathcal{O}$ ([11], Section 11). Let $\sigma(\theta)$ be the $\sigma$-field generated by $\theta$; it is essential to notice that $\sigma(\mathcal{\theta})$ is the Baire $\sigma$-field of $S$ ([5], p. 221). Because $\psi$ and $\psi^{-1}$ take Baire sets to Baire sets, $U$ can be extended uniquely to a $\sigma$-automorphism of $\sigma(\mathcal{O}) . \mathscr{I}(\mathcal{F})$ and $\mathcal{E}(\mathcal{F})$ are in obvious 1-1 correspondence with $\mathscr{T}(\mathcal{O})$ and $\mathcal{E}(\mathcal{O})$ respectively. Each finitely additive probability $\eta$ on $\theta$ is in fact countably additive, and so admits unique extension to a countably additive probability on $\sigma(\mathcal{\theta})$. To see this, it is enough to demonstrate that $B_{1}, B_{2}, \cdots$ in $\Theta$ and $B_{1} \supset B_{2} \supset \cdots, \bigcap_{i=1}^{\infty} B_{i}=\varnothing$ imply $\eta\left(B_{i}\right) \rightarrow 0$ ([5], pp. 39 and 54). But this condition is trivially satisfied, for since the $B$ 's are compact and have void intersection, some $B_{i}$ is already void.

Recall that if $\eta$ is any countably additive probability on $\sigma(\mathcal{\theta})$ and for $A, B \varepsilon \sigma(\mathcal{O})$, the distance from $A$ to $B$ is defined to be $\eta(A \triangle B)$, then $\sigma(\mathcal{O})$ is a complete semi-metric space, and moreover that every member of the space is the limit of a Cauchy sequence of elements of $\theta$. Easy consequences of these facts are as follows: (a) $\mathscr{T}(\mathcal{O})=\mathscr{T}(\sigma(\mathcal{O}))$; (b) if $\nu \varepsilon \mathscr{G}(\sigma(\mathcal{O}))$ then $\nu \varepsilon \mathcal{E}(\mathcal{O})$ iff there does not exist $B \varepsilon \sigma(\mathcal{O})$ for which $\nu(B \triangle U B)=0,0<\nu(B)<1$. The existence of such a $B$ implies that of a $C$ satisfying $U C=C, 0<\nu(C)<1$-for example, take $C=\lim \sup U^{n} B$.

An argument given by Choquet (see [7], pp. 81-2) and (a) imply $\mathfrak{I}(\mathfrak{F})$ is nonempty. Briefly, the set of all finite, signed, countably additive Baire measures on $S$ can be viewed as the dual space of the Banach space of continuous, realvalued functions on $S$. With those measures given the weak ${ }^{*}$ topology, the maps $U^{n}, n=0, \pm 1, \pm 2, \cdots$ induce continuous linear transformations of the (compact, convex) set of countably additive probabilities, $\mathcal{\odot}$, onto itself. And $\mathscr{g}(\sigma(\mathcal{\theta}))$ is the subset of $\beta$ consisting of fixed points for the maps $U^{n}$. The MarkovKakutani fixed point theorem ([3], p. 456) implies $\mathscr{g}(\sigma(\vartheta))$ is not empty, and hence that $\mathscr{I}(\mathcal{F})$ is not empty.

In view of the existence of $\psi,(a)$, the remark following (b), and a theorem of Farrell ([4], p. 460), for each $\mu \varepsilon \mathfrak{I}(\mathcal{O})$ there exists a unique (countably additive) probability $\tilde{\lambda}$ on the $\sigma$-field of subsets of $\varepsilon(\theta)$ generated by sets of the form $\{\eta \mid \eta \varepsilon \&(\Theta), \eta(C) \leqq \alpha ; 0 \leqq \alpha \leqq 1, C \varepsilon \sigma(\vartheta)\}$ satisfying

$$
\mu(B)=\int_{\varepsilon(\vartheta)} \eta(B) d \tilde{\lambda}(\eta)
$$

for each $B \varepsilon \sigma(\mathcal{O})$. In particular (3) holds for each $B \varepsilon \mathcal{O}$. Yet for such $B, \eta(B)$ viewed as a function of $\eta \varepsilon \varepsilon(\Theta)$ is measurable with respect to the $\sigma$-field $B$ generated by sets of the form $\{\eta \mid \eta \varepsilon \mathcal{E}(\mathcal{O}), \eta(C) \leqq \alpha ; 0 \leqq \alpha \leqq 1, C \varepsilon \mathcal{O}\}$. Thus for $B \varepsilon \mathcal{O}, \tilde{\lambda}$ can be cut down to, $\widetilde{\beta}$ and (3) still holds. But now (1) follows in 
view of the correspondence between $\widetilde{B}$ and $\mathscr{B}, \mathscr{I}(\mathcal{O})$ and $\mathscr{T}(\mathcal{F})$, and $\mathcal{E}(\mathcal{O})$ and $\mathcal{E}(\mathcal{F})$.

Making use of a familiar countably additive result ([9], p. 110) it is not difficult to show that $\mathcal{E}(\mathcal{F})$ consists of those $\mu$ for which $\mu\left(A \cap T^{-j}(B)\right.$ is Cesáro summable to $\mu(A) \mu(B)$ for every $A, B \varepsilon \mathcal{F}$.

Of course the definition of $\mathcal{E}(\mathcal{F})$ is contrived so that $\mathcal{E}(\mathcal{F})$ corresponds to countably additive probabilities on $\sigma(\Theta)$ which are ergodic in the usual way. And it is implicit in (2) that $\mathcal{E}(\mathfrak{F})$ is the set of extreme points of $\mathscr{I}(\mathfrak{F})$. Yet it may be of interest to see what becomes of other definitions of ergodicity when applied to $(\Omega, F)$. For example, $\mu \varepsilon \mathcal{I}(\mathfrak{F})$ might be called ergodic if there does not exist $A \varepsilon \mathcal{F}$ for which $A=T^{-1} A, 0<\mu(A)<1$. That this would have been ridiculous is clear from the following example. Let $\Omega$ be the set of bilateral sequences of 0 's and 1's, $\mathfrak{F}$ be the smallest field containing the cylinders, and $T$ be the shift. It is easy to verify that $\Omega$ and $\varnothing$ are the only members of $\mathcal{F}$ invariant under $T^{-1}$, and so every member of $\mathfrak{g}(\mathfrak{F})$ would be ergodic by this definition. (With $\Omega$ given the ordinary product topology, $\mathcal{F}$ is a base of compact open sets, and so $\Omega$ is its own Stone space.

It may seem reasonable to call $\mu \varepsilon \mathcal{G}(\mathfrak{F})$ ergodic if there does not exist $B \in \mathcal{F}$ for which $\mu\left(B \triangle T^{-1} B\right)=0,0<\mu(B)<1$. Unlike the case with $\sigma(\Theta)$ and $U$, this definition is not identical to the one just discussed, and it is true that a $\mu$ not ergodic in this sense is not ergodic according to the definition adopted. Probabilities ergodic in this sense need not be extreme in $\mathscr{T}(\mathfrak{F})$, however, as is illustrated by an example. Let $(\Omega, \mathcal{F})$ be as in the previous paragraph. Suppose $\mu$ is a measure on $\mathcal{F}$ induced by any sequence of exchangeable random variables which are neither independent nor identically 1 with probability $\alpha$ and identically 0 with probability $1-\alpha$ for some positive $\alpha<1$. Then it follows from deFinetti's theorem ([2], Chapter 4; [6], p. 486) that the only sets $B \varepsilon \mathcal{F}$ satisfying $\mu\left(B \triangle T^{-1} B\right)=0$ are $\Omega$ and $\varnothing$, and that $\mu$ is not extreme in the exchangeable probabilities on $\mathfrak{F}$, let alone in $\mathfrak{T}(\mathfrak{F})$.

3. Generalizations. Apparently several times in Section $2 T^{-1}$ or $\psi^{-1}$ was considered when reference to the inverse was unnecessary. The reason for this was to keep notation consistent with that of more general problems than the one being studied. What follows is an outline of several results similar to (1), some generalizations and some not comparable. The interested reader will have no trouble filling in the details himself.

Suppose $\Omega$ is a set, $\mathfrak{F}$ a field of subsets, and $\mathfrak{J}$ a family of $\mathcal{F}$-measurable maps of $\Omega$ into itself. Then $T \& \mathcal{J}$ implies $T^{-1}$ can be viewed as a homomorphism of $\mathfrak{F}$ (though the same is not necessarily true of $T$ ). A finitely additive probability $\mu$ on $\mathcal{F}$ is in $\mathscr{I}(\mathcal{F})$ if $\mu(A)=\mu\left(T^{-1} A\right)$ for each $T \varepsilon \mathcal{J}$ and $A \varepsilon \mathcal{F}$; it is in $\mathcal{E}(\mathcal{F})$ if there do not exist $\delta>0$ and $A_{1}, A_{2}, \cdots \varepsilon \mathcal{F}$ for which $\delta<\mu\left(A_{n}\right)<$ $1-\delta, \lim _{m, n \rightarrow \infty} \mu\left(A_{n} \triangle A_{m}\right)=0$, and $\mu\left(A_{n} \triangle T^{-1} A_{n}\right) \rightarrow 0$ for each $T \varepsilon \mathfrak{\xi}$.

Again the Stone space argument can be employed. If $U$ on $\mathcal{O}$ corresponds to $T^{-1}, T \in$ J, there may not be a homeomorphism of the Stone space corresponding 
to $U$, but at least there is a unique continuous map $\xi$ of $S$ into $S$ satisfying: $U B=\xi^{-1} B$ for each $B \varepsilon \Theta([11]$, p. 32$)$. Hence the results of Farrell ([4], p. 460) and Choquet and Feldman ([7], pp. 82-3) apply to yield a number of theorems similar to (1). Of course, that $\mathscr{g}(\mathfrak{F})$ is not empty does not follow from the MarkovKakutani fixed point theorem unless the maps in $J$ commute under composition. Yet other fixed point theorems can be employed in specific situations (see [8], Section 5; [3], p. 457).

With the definition of crgodicity given in this section, every member of $\mathcal{E}(\mathcal{F})$ is an extreme point of $\mathscr{g}(\mathfrak{F})$. And the present definition is analogous to that of Phelps ([7], p. 81). Farrell's definition would have $\mu \varepsilon \mathscr{J}(\mathcal{F})$ ergodic if there does not exist $B \varepsilon \sigma(\theta)$ satisfying both $B=U B$ for each $U$ on $\sigma(\theta)$ (corresponding to $a T^{-1}$ ) and $0<\nu(B)<1$, where $\nu$ on $\sigma(\mathcal{O})$ corresponds to $\mu \varepsilon \mathfrak{I}(\mathcal{F})$. For conditions that a $\mu$ ergodic in Farrell's sense be in $\mathcal{E}(\mathcal{F})$ see ([4], p. 452; [12], pp. 196-7). In general his definition is not equivalent to either definition mentioned in the last section.

It seems pointless to present a catalogue of representation theorems including results on the nonemptiness of $\mathscr{G}(\mathfrak{F})$ and the uniqueness of the representation. But perhaps one striking fact deserves mention. Namely, the existence of a representation of the form (2) requires not only no assumptions on $\Omega$ and $\mathcal{F}$, but also no assumptions on the maps $\mathcal{I}$ other than their measurability. The only qualification is this. If $\mathcal{E}(\mathcal{\theta})$ is not weak ${ }^{*}$ closed in $\mathscr{J}(\sigma(\mathcal{O}))$, the $\sigma$-field $\Theta$ in (1) must be enlarged in a manner similar to that described by Bishop and deLeenw (see [7], pp. 31 and $\$ 3 ;$ [6], p. 481).

4. An interpretation. One reason for being interested in finitely additive probabilities is a sympathy with the notion of subjective probability ([2]; [10]). Thus it may seem interesting that both invariance and ergodicity admit subjective interpretation. For purposes of illustration, assume again that $\Omega, F$, and $T$ are as in the examples of Section 2. Each $\mu$ on $\mathfrak{F}$ determines a law for the coordinate process $X_{n}(\omega)=\omega(n)$, the $n$th coordinate of $\omega$. If $\mu$ represents your beliefs about $X_{n}$, and $n$ represents time, then $\mu$ is invariant for you if the probability of any pattern of 0 's and 1's of finite length is invariant under shifts in the time label. $\mu$ is ergodic if, for you, the $\lambda$ of (2) is a point mass (cf. [10], p. 53). The frequentistic notion of ergodicity ([9], pp. 104-5) involving averages figuring in the ergodic theorem does not fit comfortably into the framework of subjective probability (cf. [2], Chapter VI; [10], Chapter 3).

\section{REFERENCES}

[1] CA'LER, ‥ (1961). Sume representation theorems for invariant probability measures. Illinois .J. Math. 8 408-418.

[2] nEFinetri, B. (1937). La prevision: ses lois logiques, ses sources subjectives. Ann. Inst. H. Poincaré 7 1-68. 'Translated by H. Kyburg and reprinted with annotations by deFinetti in Studies in Subjective Probability, H. Kyburg, Jr. and H. Smokler (editors), Wiley, 1964.)

[3] Dunford, N. and Scrwintz, J. T. (1958). Linear Operators, Part I. Interscience, New York. 
[4] FARRELL, R. H. (1962). Representation of invariant measures. Illinois J. Mulh. $6417-$ 467.

[5] Halmos, P. R. (1950). Measure Theory. Van Nostrand, Princeton.

[6] Hewitt, E. and Savage, L. J. (1955). Symmetric measures on cartesian products. Trans. Amer. Malh. Soc. 80 470-501.

[7] Phelps, R. R. (1966). Lectures on Choquet's T'heorem. Van Nostrand, Princeton.

[8] Rickert, N. W. (1967). Amenable groups and groups with the fixed point property. Trans. Amer. Math. Soc. 127 221-232.

[9] Rosenblatt, M. (1962). Random Processes. Oxford Univ. Press, New York.

[10] Savage, L. J. (1954). The Foundations of Statistics. Wiley, New York.

[11] Sikorski, R. (1960). Boolean Algebras. Springer, Berlin.

[12] Varadarajan, V. S. (1963). Groups of antomorphisms of Borel spaces. Trans. Amer. Math. Soc. 109 191-220. 